\title{
A regularity result for a class of elliptic equations with lower order terms
}

\section{Claudia Capone ${ }^{1} \cdot$ Teresa Radice $^{2}$ (D)}

Received: 20 June 2020 / Accepted: 20 July 2020 / Published online: 6 August 2020

(c) The Author(s) 2020

\section{Abstract}

In this paper we establish the higher differentiability of solutions to the Dirichlet problem

$$
\begin{cases}\operatorname{div}(A(x, D u))+b(x) u(x)=f & \text { in } \Omega \\ u=0 & \text { on } \partial \Omega\end{cases}
$$

under a Sobolev assumption on the partial map $x \rightarrow A(x, \xi)$. The novelty here is that we take advantage from the regularizing effect of the lower order term to deal with bounded solutions.

Keywords A priori estimate $\cdot$ Boundedness of solution $\cdot$ Regularizing effect $\cdot$ Approximation

Mathematics Subject Classification 35B65 · 35J60 - 49N60 • 49N99

\section{Introduction}

This paper concerns the higher differentiability and the higher integrability of the gradient of local weak solution of the Dirichlet problem

$$
\begin{cases}\operatorname{div}(A(x, D u))+b(x) u(x)=f & \text { in } \Omega \\ u=0 & \text { on } \partial \Omega\end{cases}
$$

Teresa Radice

teresa.radice@unina.it

Claudia Capone

capone@na.iac.cnr.it

1 Istituto per le Applicazioni del Calcolo "Mauro Picone", sezione di Napoli, Consiglio Nazionale delle Ricerche, via Pietro Castellino 111, 80131 Napoli, Italy

2 Dipartimento di Matematica e Applicazioni “R. Caccioppoli”, Università degli Sudi di Napoli "Federico II", Via Cintia, 80126 Napoli, Italy 
The operator $A: \Omega \times \mathbb{R}^{n} \rightarrow \mathbb{R}^{n}$ is a Carathéodory mapping, satisfying for positive constants $\alpha, \beta>0$ the following assumptions

$$
\begin{gathered}
\alpha|\xi-\eta|^{2} \leqslant\langle A(x, \xi)-A(x, \eta), \xi-\eta\rangle, \\
|A(x, \xi)-A(x, \eta)| \leqslant \beta|\xi-\eta| .
\end{gathered}
$$

Concerning the dependence on $x$-variable, it is clear that no extra differentiability can be obtained for solutions even if the data $f(x)$ and $b(x)$ are smooth, unless some differentiability assumption is made on the operator $A(x, \xi)$.

To this aim, we shall assume that there exists a non negative function $k(x) \in L_{l o c}^{n}(\Omega)$, such that

$$
|A(x, \xi)-A(y, \xi)| \leqslant(k(x)+k(y))|x-y|(1+|\xi|)
$$

for every $\xi \in \mathbb{R}^{n}$ and a.e. $x, y \in \Omega$. Finally, we shall assume

$$
|f(x)| \leqslant Q b(x)
$$

for a.e. $x \in \Omega$.

Conditions (1.2) and (1.3) express the uniform ellipticity and lipschitz continuity of the operator $A(x, \xi)$ with respect to the variable $\xi$.

Condition (1.4), in view of the pointwise characterization of the Sobolev spaces [12], means that the partial map $x \rightarrow A(x, \xi)$ belongs to the Sobolev class $W_{l o c}^{1, n}(\Omega)$.

Finally, condition (1.5), introduced in Ref. [1] (see also Ref. [2]), relates the coefficient of the lower order term with the right hand side.

This interplay yields a regularizing effect on the solution of the Dirichlet problem (1.1). More precisely, it is sufficient to assume (1.5) to obtain

$$
\|u\|_{L^{\infty}(\Omega)} \leqslant Q .
$$

The key tool to deal with equations with lower order terms, assuming a low integrability for $b(x)$ and $f(x)$ as in (1.1), is the result in Ref. [1] (see also Ref. [2]).

Recall that the boundedness of the solution of equation (1.1) is well known if $b(x), f(x) \in L^{s}(\Omega)$ for some $s>\frac{n}{2}[10]$, and usually it is the first step in the analysis of the regularity of the solutions and open the way to the investigation of some higher regularity for the solutions.

We would like to mention that, under $L^{1}$ integrability on the right hand side $f$, existence and uniqueness of the solutions have been established in Ref. [7], when $Q=0$.

Recent works have shown that the $W^{1, n}$ - regularity of the map $x \rightarrow A(x, \xi)$ is sufficient to obtain the higher differentiability of the solutions of the problem (1.1) but in the case $b=f=0$ (see Refs. [5, 8, 9, 16, 17]).

The above mentioned results hold true also for the so-called $p$-harmonic operator, as well as for local minimizers of integral functionals with $p$-growth.

Here we take advantage from the result in Ref. [2] to deal with equation as in (1.1) and prove the following 
Theorem 1 Let $u \in W_{0}^{1,2}(\Omega)$ be the solution of the Dirichlet problem (1.1). Assume that $A(x, \xi)$ satisfy (1.2)- (1.4), that $b(x) \in L^{2}(\Omega)$ and that (1.5) is satisfied for some constant $Q>0$. Then $u \in W_{\text {loc }}^{2,2}(\Omega)$ and the following second order Caccioppoli type inequality

$$
\int_{B_{\frac{R}{2}}}\left|D^{2} u\right|^{2} \leqslant \frac{C(\alpha, \beta, Q)}{R^{2}}\left(\int_{B_{R}}|D u|^{2}+\int_{B_{R}}|b|^{2}\right),
$$

holds for every ball $B_{R} \subset \Omega$.

We would like to pointout that we only require $b(x), f(x) \in L^{2}(\Omega)$ and this assumption, which is stronger than the one used in Ref. [2], is still weaker than the classical one in order to deal with bounded solution for $n \geq 4$. On the other hand, in order to obtain higher differentiability for solutions to problem (1.1), condition (1.5) cannot be dropped without assuming higher integrability on $b(x)$ and $f(x)$.

Indeed, condition (1.5) is essential to have Theorem 1, under the hypothesis $b(x), f(x) \in L^{2}$ (see example in Section 4).

On the other hand, it is easy to check that Theorem 1 still holds true without condition (1.5), but assuming $b(x) \in L^{n}$.

The proof of Theorem 1 is obtained combining a suitable a priori estimate for the second derivatives of the solution of the equation with an approximation argument.

To establish the a priori estimate for the solution to (1.1) which has discontinuous coefficients we use the classical tool of the difference quotient method.

The main difficulty in establishing the a priori estimate is that we need to deal with terms with critical integrability that have to be reabsorbed and we shall succeed by suitable iteration argument.

Next, we use an approximation procedure that is constructed by introducing Dirichlet problems that, on one hand, admit solutions with the second derivative in $L_{l o c}^{2}(\Omega)$ and, on the other, satisfy the assumptions of the result in Ref. [1]. Also we need to prove that the solutions of each approximating Dirichlet problem are uniformly bounded by the same constant $Q$ appearing in assumption (1.4).

As a consequence of our higher differentiability result combined with the boundedness of $u$ we also have the following higher integrability for the gradient of the solutions

Corollary 1 Let $u \in W_{0}^{1,2}(\Omega)$ be the solution of the Dirichlet problem (1.1). Assume that $A(x, \xi)$ satisfy (1.2)- (1.4) and $b(x) \in L^{2}(\Omega)$ and that (1.5) is satisfied for some constant $Q>0$. Then $D u \in L_{\text {loc }}^{4}(\Omega)$ and the following estimate

$$
\int_{B_{\frac{R}{2}}}|D u|^{4} \leqslant C\left(\int_{B_{R}}\left(1+|D u|^{2}+b^{2}\right)\right)
$$

holds, for a constant $C=C(Q, R)$. 
Such higher integrability result is a consequence of a suitable Gagliardo Nirenberg type inequality and it is inspired by the arguments introduced in Ref. [6] (for other Gagliardo Nirenberg type inequalities see also Ref. [4]). It is well known that the $W^{1, n}$ -regularity of the coefficients has been employed in the study of non variational equations $[3,15]$. Note that $W^{1, n} \subset V M O \subset B M O$, variational and non variational equations under $V M O$ and $B M O$ coefficients have been widely investigated. Among the others, we quote the results in Refs. [11, 13, 14, 18-20].

Obviously, once the existence and the $W^{2,2}$-regularity of the solution of (1.1) has been proven, the solution $u$ of the equation in (1.1) is also a solution to a non variational equation. In a forthcoming project we shall examine the analogous problem for the non variational case with lower order terms.

\section{Notations and preliminary results}

In Ref. [1] the authors studied boundedness of the solutions of the following problem:

$$
\begin{cases}-\operatorname{div}(A(x, \nabla u))+a(x) u|u|^{p-2}=f(x) & \text { in } \Omega \\ u=0 & \text { on } \partial \Omega\end{cases}
$$

with $2<p \leqslant n$ and $A: \Omega \times \mathbb{R}^{n} \rightarrow \mathbb{R}^{n}$ is a Carathéodory function satisfying for some positive constants $\alpha, \beta$ and a function $h(x) \in L^{p^{\prime}}(\Omega)$ that

$$
\begin{gathered}
A(x, \xi) \xi \geqslant \alpha|\xi|^{p}, \\
|A(x, \xi)| \leqslant h(x)+\beta|\xi|^{p-1},
\end{gathered}
$$

and

$$
(A(x, \xi)-A(x, \eta))(\xi-\eta)>0, \quad \xi \neq \eta
$$

for almost every $x \in \Omega$, for every $\xi$ and $\eta \in \mathbb{R}^{n}$ and

$$
|f(x)| \leqslant Q a(x)+R(x) .
$$

In Ref. [1] it has been proven that $u \in L^{\infty}(\Omega)$. More precisely,

Theorem 2 If $a(x), \quad f(x) \in L^{1}(\Omega)$ with $a(x) \geqslant 0$ and $0 \leqslant R(x) \in L^{\left(p^{\star}\right)^{\prime}}$ satisfy (2.5) and if (2.2), (2.3), (2.4) hold then there exists a unique weak solution $u \in W_{0}^{1, p}(\Omega) \cap L^{\infty}(\Omega)$ of $(2.1)$.

We will use previous theorem in the case $p=2$ and with $h(x)=0=R(x)$. In this particular case, the precise bound for the $L^{\infty}$-norm of $u$, which will be used in the sequel, is the following

$$
\|u\|_{L^{\infty}(\Omega)} \leqslant Q
$$




\subsection{Difference quotient}

In order to get the higher differentiability of the solutions (1.1) we will use the difference quotient method. Therefore in this section we introduce the finite difference operator and we recall the basic properties.

Definition 1 For every vector valued function $F: \mathbb{R}^{n} \rightarrow \mathbb{R}^{N}$ the finite difference operator is defined by

$$
\tau_{s, h} F(x)=F\left(x+h e_{s}\right)-F(x)
$$

where $h \in \mathbb{R}, e_{s}$ is the unit vector in the $x_{s}$ direction and $s \in\{1, \ldots, n\}$.

The difference quotient is defined for $h \in \mathbb{R} \backslash\{0\}$ as

$$
\Delta_{s, h} F(x)=\frac{\tau_{s, h} F(x)}{h} .
$$

The following proposition describes some elementary properties of the finite difference operator and can be found, for example, Ref. [10].

Proposition 1 Let $f$ and $g$ be two functions such that $F, G \in W^{1, p}\left(\Omega ; \mathbb{R}^{N}\right)$, with $p \geq 1$, and let us consider the set

$$
\Omega_{|h|}:=\{x \in \Omega: \operatorname{dist}(x, \partial \Omega)>|h|\} .
$$

Then

(d1) $\tau_{s, h} F \in W^{1, p}(\Omega)$ and

$$
D_{i}\left(\tau_{s, h} F\right)=\tau_{s, h}\left(D_{i} F\right) .
$$

(d2) If at least one of the functions $F$ or $G$ has support contained in $\Omega_{|h|}$ then

$$
\int_{\Omega} F \tau_{s, h} G d x=-\int_{\Omega} G \tau_{s,-h} F d x
$$

(d3) We have

$$
\tau_{s, h}(F G)(x)=F\left(x+h e_{s}\right) \tau_{s, h} G(x)+G(x) \tau_{s, h} F(x) .
$$

The next result about finite difference operator is a kind of integral version of Lagrange Theorem.

The following results will be useful in the sequel

Lemma 1 If $0<\rho<R,|h|<\frac{R-\rho}{2}, 1<p<+\infty, s \in\{1, \ldots, n\}$ and $F, D_{s} F \in L^{p}\left(B_{R}\right)$ then 


$$
\int_{B_{\rho}}\left|\tau_{s, h} F(x)\right|^{p} d x \leq|h|^{p} \int_{B_{R}}\left|D_{s} F(x)\right|^{p} d x .
$$

Moreover

$$
\int_{B_{\rho}}\left|F\left(x+h e_{s}\right)\right|^{p} d x \leq c(n, p) \int_{B_{R}}|F(x)|^{p} d x .
$$

Now, we recall the fundamental Sobolev embedding property (for the proof we can refer, for example [10] [Lemma 8.2]).

Lemma 2 Let $F: \mathbb{R}^{n} \rightarrow \mathbb{R}^{N}, F \in L^{p}\left(B_{R}\right)$ with $1<p<+\infty$. Suppose that there exist $\rho \in(0, R)$ and $M>0$ such that

$$
\sum_{s=1}^{n} \int_{B_{\rho}}\left|\tau_{s, h} F(x)\right|^{p} d x \leq M^{p}|h|^{p},
$$

for every $h$ with $|h|<\frac{R-\rho}{2}$. Then $F \in W^{1, p}\left(B_{\rho} ; \mathbb{R}^{N}\right) \cap L^{\frac{n p}{n-p}}\left(B_{\rho} ; \mathbb{R}^{N}\right)$. Moreover

$$
\|\left. D F\right|_{L^{p}\left(B_{\rho}\right)} \leq M
$$

and

$$
\|F\|_{L^{\frac{n p}{n-p}\left(B_{\rho}\right)}} \leq c\left(M+\|F\|_{L^{p}\left(B_{R}\right)}\right),
$$

with $c \equiv c(n, N, p)$.

An iteration Lemma finds an important application in the so called hole-filling method. A proof can be found, for example, Ref. [10] (see Lemma 6.1).

Lemma 3 Let $h:\left[\rho, R_{0}\right] \rightarrow \mathbb{R}$ be a non-negative bounded function and $0<\vartheta<1$, $A, B \geq 0$ and $\beta>0$. Assume that

$$
h(r) \leq \vartheta h(d)+\frac{A}{(d-r)^{\beta}}+B
$$

for all $\rho \leq r<d \leq R_{0}$. Then

$$
h(\rho) \leq \frac{c A}{\left(R_{0}-\rho\right)^{\beta}}+B
$$

where $c=c(\vartheta, \beta)>0$.

The following Lemma is a sort of Gagliardo Nirenberg interpolation inequality Lemma 4 For $\Psi \in C_{c}^{1}(\Omega)$ with $\Psi \geqslant 0$ and $C^{2}$ maps $v: \Omega \rightarrow \mathbb{R}^{n}$ we have 


$$
\begin{aligned}
& \int_{\Omega} \Psi^{\frac{m}{m+1}}{ }^{(p+2)}|D \nu|^{\frac{m}{m+1}(p+2)} d x \leqslant(p+2)^{2}\left(\int_{\Omega} \Psi^{\frac{m}{m+1}(p+2)}|v|^{2 m} d x\right)^{\frac{1}{m+1}} \\
& \times\left[\left(\int_{\Omega} \Psi^{\frac{m}{m+1}(p+2)}|D \Psi|^{2}|D v|^{p} d x\right)^{\frac{m}{m+1}}+n\left(\int_{\Omega} \Psi^{\frac{m}{m+1}(p+2)}|D v|^{p-2}\left|D^{2} v\right|^{2} d x\right)^{\frac{m}{m+1}}\right]
\end{aligned}
$$

where $p \in(1, \infty)$ and $m>1$. Moreover

$$
\begin{aligned}
& \int_{\Omega} \Psi^{2}\left(1+|D v|^{2}\right)^{\frac{p}{2}}|D v|^{2} d x \leqslant c(p)\|v\|_{L^{\infty}(\operatorname{supp} \Psi)}^{2} \int_{\Omega} \Psi^{2}\left(1+|D v|^{2}\right)^{\frac{p-2}{2}}\left|D^{2} v\right|^{2} d x \\
& \quad+c\|v\|_{L^{\infty}(\operatorname{supp} \Psi)} \int_{\Omega}\left(|\Psi|^{2}+|\nabla \Psi|^{2}\right)\left(1+|D v|^{2}\right)^{\frac{p}{2}} d x
\end{aligned}
$$

for a positive constant $c=c(p)$.

(For the proof we refer to Refs. [6] and [9]).

\section{Proof of Theorem 1}

This section is devoted to the proof of our main result.

We shall divide it in two steps: in the first one we establish an a priori estimate for the second derivatives of the solutions and in the second one we construct the suitable approximating problems and we shall prove that the a priori estimate is preserved in passing to the limit.

\section{Proof Step 1. The a priori estimate}

Suppose that $u$ is a local solution of the problem (1.1) such that $u \in W_{0}^{1,2}(\Omega) \cap W_{l o c}^{2,2}(\Omega)$.

Let us fix a ball $B_{R} \subset \Omega$ and arbitrary radii $\frac{R}{2}<r<\tilde{s}<t<\lambda r<R$, with $\lambda \in(1,2)$. Consider a cut-off function $\eta \in C_{0}^{\infty}\left(B_{t}\right)$ such that $\eta \equiv 1$ on $B_{\tilde{s}}$ and $|D \eta| \leq \frac{c}{t-\tilde{s}}$. Using $\varphi=\tau_{s,-h}\left(\eta^{2} \tau_{s, h} u\right)$ as a test function in the equation in (1.1) we get

$$
\begin{aligned}
& \int_{B_{t}} A(x, D u) D\left(\tau_{s,-h}\left(\eta^{2} \tau_{s, h} u\right)\right)+\int_{B_{t}} b(x) u(x) \tau_{s,-h}\left(\eta^{2} \tau_{s, h} u\right) \\
& =\int_{B_{t}} f(x) \tau_{s,-h}\left(\eta^{2} \tau_{s, h} u\right) .
\end{aligned}
$$

By $(d 1),(d 2)$ of Proposition 1, the first integral in the left hand side in (3.1) can be written as follows:

$$
\begin{aligned}
& \int_{B_{t}} A(x, D u) D\left(\tau_{s,-h}\left(\eta^{2} \tau_{s, h} u\right)\right)=\int_{B_{t}} A(x, D u) \tau_{s,-h}\left(D\left(\eta^{2} \tau_{s, h} u\right)\right) \\
& =-\int_{B_{t}} \tau_{s, h}(A(x, D u)) D\left(\eta^{2} \tau_{s, h} u\right) .
\end{aligned}
$$


Inserting (3.2) in (3.1)

$$
\begin{aligned}
& \int_{B_{t}} \tau_{s, h}(A(x, D u)) D\left(\eta^{2} \tau_{s, h} u\right)=\int_{B_{t}} b(x) u(x) \tau_{s,-h}\left(\eta^{2} \tau_{s, h} u\right) \\
& -\int_{B_{t}} f(x) \tau_{s,-h}\left(\eta^{2} \tau_{s, h} u\right) .
\end{aligned}
$$

On the other hand, the left hand side of (3.3) can be written as follows

$$
\begin{aligned}
\int_{B_{t}} \tau_{s, h}(A(x, D u)) D\left(\eta^{2} \tau_{s, h} u\right) \\
=\int_{B_{t}} \tau_{s, h}(A(x, D u))\left(2 \eta \tau_{s, h} u D \eta+\eta^{2} \tau_{s, h} D u\right) \\
=\int_{B_{t}}\left\langle A(x+h, D u(x+h))-A(x+h, D u(x)), \eta^{2} \tau_{s, h} D u\right\rangle \\
\quad+\int_{B_{t}}\left\langle A(x+h, D u(x))-A(x, D u(x)), \eta^{2} \tau_{s, h} D u\right\rangle \\
\quad+2 \int_{B_{t}}\left\langle A(x+h, D u(x+h))-A(x+h, D u(x)), \eta \tau_{s, h} u D \eta\right\rangle \\
\quad+2 \int_{B_{t}}\left\langle A(x+h, D u(x))-A(x, D u(x)), \eta D \eta \tau_{s, h} u\right\rangle .
\end{aligned}
$$

Substituting in (3.3) we obtain:

$$
\begin{aligned}
\int_{B_{t}}\left\langle A(x+h, D u(x+h))-A(x+h, D u(x)), \eta^{2} \tau_{s, h} D u\right\rangle \\
=\int_{B_{t}}\left\langle A(x, D u(x))-A(x+h, D u(x)), \eta^{2} \tau_{s, h} D u\right\rangle \\
\quad-2 \int_{B_{t}} \eta\left\langle A(x+h, D u(x+h))-A(x+h, D u(x)), D \eta \tau_{s, h} u\right\rangle \\
\quad-2 \int_{B_{t}} \eta\left\langle A(x+h, D u(x))-A(x, D u(x)), D \eta \tau_{s, h} u\right\rangle \\
\quad+\int_{B_{t}} b(x) u(x)\left(\tau_{s,-h}(\eta) \eta \tau_{s, h} u+\eta \tau_{s,-h}\left(\eta \tau_{s, h} u\right)\right) \\
-\int_{B_{t}} f(x)\left(\tau_{s,-h}(\eta) \eta \tau_{s, h} u+\eta \tau_{s,-h}\left(\eta \tau_{s, h} u\right)\right) .
\end{aligned}
$$

Using assumptions (1.3), (1.4), (1.5) in the right hand side of the previous estimate, we get 


$$
\begin{aligned}
& \int_{B_{t}}\left\langle A(x+h, D u(x+h))-A(x+h, D u(x)), \eta^{2} \tau_{s, h} D u\right\rangle \\
& \quad \leqslant|h| \int_{B_{t}} \eta^{2}(k(x)+k(x+h))(1+|D u|)\left|\tau_{s, h} D u\right| \\
& \quad+\beta \int_{B_{t}}|D \eta||\eta||D u(x+h)-D u(x)|\left|\tau_{s, h} u\right| \\
& \quad+2|h| \int_{B_{t}} \eta|D \eta|(k(x)+k(x+h))(1+|D u|)\left|\tau_{s, h} u\right| \\
& \quad+\int_{B_{t}} b(x)\left|\tau_{s,-h}(\eta) \eta \tau_{s, h} u\right||u|+\int_{B_{t}} b(x)\left|\eta \tau_{s,-h}\left(\eta \tau_{s, h} u\right)\right||u| \\
& \quad+Q \int_{B_{t}} b(x)\left|\tau_{s,-h}(\eta) \eta \tau_{s, h} u\right|+Q \int_{B_{t}}\left|b(x) \eta \tau_{s,-h}\left(\eta \tau_{s, h} u\right)\right| .
\end{aligned}
$$

Since the assumptions of Theorem 2 are satisfied, we can use that $\|u\|_{L^{\infty}(\Omega)} \leqslant Q$ thus getting

$$
\begin{aligned}
\int_{B_{t}}\left\langle A(x+h, D u(x+h))-A(x+h, D u(x)), \eta^{2} \tau_{s, h} D u\right\rangle \\
\leqslant|h| \int_{B_{t}} \eta^{2}(k(x)+k(x+h))(1+|D u|)\left|\tau_{s, h} D u\right|+\beta \int_{B_{t}} \eta\left|\tau_{s, h} D u\right|\left|\tau_{s, h} u\right||D \eta| \\
\quad+2|h| \int_{B_{t}} \eta|D \eta|(k(x)+k(x+h))(1+|D u|)\left|\tau_{s, h} u\right| \\
\quad+2 Q \int_{B_{t}} b(x)\left|\tau_{s,-h}(\eta) \eta \tau_{s, h} u\right|+2 Q \int_{B_{t}} \eta b(x)\left|\tau_{s,-h}\left(\eta \tau_{s, h} u\right)\right| .
\end{aligned}
$$

Using the ellipticity assumption (1.2) in the left hand side and Young's inequality in the right hand side of (3.4), since $\eta \in C_{0}^{\infty}\left(B_{t}\right)$, by Lagrange Theorem, the previous inequality gives: 


$$
\begin{aligned}
& \alpha \int_{B_{t}} \eta^{2}\left|\tau_{s, h} D u\right|^{2} \leqslant C_{\varepsilon}|h|^{2} \int_{B_{t}} \eta^{2}(k(x)+k(x+h))^{2}(1+|D u|)^{2}+\varepsilon \int_{B_{t}} \eta^{2}\left|\tau_{s, h} D u\right|^{2} \\
& +\varepsilon \int_{B_{t}} \eta^{2}\left|\tau_{s, h} D u\right|^{2}+c(\varepsilon, \beta) \int_{B_{t}}|D \eta|^{2}\left|\tau_{s, h} u\right|^{2} \\
& +C|h|^{2} \int_{B_{t}} \eta^{2}(k(x)+k(x+h))^{2}(1+|D u|)^{2}+C \int_{B_{t}}|D \eta|^{2}\left|\tau_{s, h} u\right|^{2} \\
& +2 Q|h| \int_{B_{t}} \eta|D \eta||b(x)|\left|\tau_{s, h} u\right|+2 Q \int_{B_{t}} \eta|b(x)|\left|\tau_{s,-h}\left(\eta \tau_{s, h} u\right)\right| \\
& \quad \leqslant C_{\varepsilon}|h|^{2} \int_{B_{t}}(k(x)+k(x+h))^{2}(1+|D u|)^{2}+2 \varepsilon \int_{B_{t}} \eta^{2}\left|\tau_{s, h} D u\right|^{2} \\
& +\frac{c(\varepsilon, \beta, Q)}{(t-\tilde{s})^{2}} \int_{B_{t}}\left|\tau_{s, h} u\right|^{2}+C(Q)|h|^{2} \int_{B_{t}}|b(x)|^{2} \\
& +2 Q \int_{B_{t}}|b(x)|\left|\tau_{s,-h}\left(\eta \tau_{s, h} u\right)\right|,
\end{aligned}
$$

where we also used that $|D \eta| \leqslant \frac{C}{t-\tilde{s}}$.

Choosing $\varepsilon=\frac{\alpha}{4}$, we can reabsorb the second integral in the right hand side of the previous estimate by the left hand side, moreover we can apply Lemma 1 and the properties of $\eta$ so as to obtain

$$
\begin{aligned}
& \frac{\alpha}{2} \int_{B_{t}} \eta^{2}\left|\tau_{s, h} D u\right|^{2} \leqslant C_{\alpha}|h|^{2} \int_{B_{t}}(k(x)+k(x+h))^{2}(1+|D u|)^{2} \\
& \quad+\frac{C(\alpha, \beta, Q)}{(t-\tilde{s})^{2}}|h|^{2} \int_{B_{\lambda r}}|D u|^{2}+C(Q)|h|^{2} \int_{B_{t}}|b(x)|^{2} \\
& \quad+2 Q \int_{B_{t}}|b(x)|\left|\tau_{s,-h}\left(\tau_{s, h} u\right)\right|
\end{aligned}
$$

Dividing both sides of previous estimate by $|h|^{2}$ we obtain that

$$
\begin{aligned}
& \frac{\alpha}{2} \int_{B_{t}} \eta^{2} \frac{\left|\tau_{s, h} D u\right|^{2}}{|h|^{2}} \leqslant\left. C_{\alpha} \int_{B_{t}}(k(x)+k(x+h))^{2}(1+\mid D u)\right|^{2} \\
& \quad+\frac{C(\alpha, \beta, Q)}{(t-\tilde{s})^{2}} \int_{B_{\lambda r}}|D u|^{2}+C(Q) \int_{B_{t}}|b(x)|^{2} \\
& \quad+2 Q \int_{B_{t}}|b(x)| \frac{\left|\tau_{s .-h}\left(\eta \tau_{s, h} u\right)\right|}{|h|^{2}} .
\end{aligned}
$$

In order to estimate the last integral of the previous inequality, we observe that 


$$
\left|\frac{\tau_{s,-h}\left(\eta \tau_{s, h} u\right)}{|h|^{2}}\right|=\frac{1}{|h|}\left|\tau_{s,-h}\left(\eta \frac{\tau_{s, h} u}{|h|}\right)\right|=\left|\frac{\tau_{s,-h}\left(\eta \frac{\tau_{s, h} u}{|h|}\right)}{h}\right| .
$$

Hence, we use Young's inequality and Lemma 1 as follows

$$
\begin{aligned}
\int_{B_{t}}|b(x)| \frac{\left|\tau_{s,-h}\left(\eta \tau_{s, h} u\right)\right|}{|h|^{2}} & =\int_{B_{t}}|b(x)|\left|\frac{\tau_{s,-h}\left(\eta \frac{\tau_{s, h} u}{|h|}\right)}{h}\right| \\
& \leqslant C_{\sigma} \int_{B_{t}}|b(x)|^{2}+\sigma \int_{B_{t}}\left|\frac{\tau_{s,-h}\left(\eta \frac{\tau_{s, h} u}{|h|}\right)}{h}\right|^{2} \\
& \leqslant C_{\sigma} \int_{B_{t}}|b(x)|^{2}+\sigma \int_{B_{t}}\left|D\left(\eta \frac{\tau_{s, h} u}{|h|}\right)\right|^{2} \\
& =C_{\sigma} \int_{B_{t}}|b(x)|^{2}+\sigma \int_{B_{t}}\left|D \eta \frac{\tau_{s, h} u}{|h|}\right|^{2}+\sigma \int_{B_{t}}\left|\eta D\left(\frac{\tau_{s . h} u}{|h|}\right)\right|^{2} \\
\leqslant & \int_{\sigma}|b(x)|^{2}+\frac{C(\sigma)}{(t-\tilde{s})^{2}} \int_{B_{\lambda r}}|D u|^{2} \\
& +\sigma \int_{B_{t}} \eta^{2} \frac{\left|\tau_{s, h} D u\right|^{2}}{|h|^{2}},
\end{aligned}
$$

for a positive $\sigma<1$, that will be chosen later.

Inserting (3.6) in (3.5)

$$
\begin{aligned}
\frac{\alpha}{2} \int_{B_{t}} \eta^{2} \frac{\left|\tau_{s, h} D u\right|^{2}}{|h|^{2}} \leqslant & C_{\alpha} \int_{B_{t}}(k(x)+k(x+h))^{2}(1+|D u|)^{2}+\frac{C(\alpha, \beta, Q)}{(t-\tilde{s})^{2}} \int_{B_{\lambda r}}|D u|^{2} \\
& +C(\sigma, Q) \int_{B_{t}}|b(x)|^{2}+\frac{C(\sigma, Q)}{(t-\tilde{s})^{2}} \int_{B_{\lambda r}}|D u|^{2} \\
& +2 \sigma Q \int_{B_{t}} \eta^{2} \frac{\left|\tau_{s, h} D u\right|^{2}}{|h|^{2}}
\end{aligned}
$$

Choosing $\sigma=\frac{\alpha}{8 Q}$, we can reabsorb the last integral in the right hand side by the left hand side, thus getting

$$
\begin{aligned}
\int_{B_{t}} \eta^{2} \frac{\left|\tau_{s, h} D u\right|^{2}}{|h|^{2}} \leqslant & C \int_{B_{t}}(k(x)+k(x+h))^{2}(1+|D u|)^{2}+\frac{C}{(t-\tilde{s})^{2}} \int_{B_{\lambda r}}|D u|^{2} \\
& +C \int_{B_{t}}|b(x)|^{2}
\end{aligned}
$$

where $C=C(\alpha, \beta, Q)$. 
Setting

$$
M=C^{\frac{1}{2}}\left[\int_{B_{t}}(k(x)+k(x+h))^{2}(1+|D u|)^{2}+\frac{1}{(t-\tilde{s})^{2}} \int_{B_{\lambda r}}|D u|^{2}+\int_{B_{t}}|b(x)|^{2}\right]^{\frac{1}{2}},
$$

inequality (3.7) becomes

$$
\int_{B_{t}} \eta^{2} \frac{\left|\tau_{s, h} D u\right|^{2}}{|h|^{2}} \leqslant M^{2}
$$

Since $\eta=1$ on $B_{\tilde{s}}$, we get

$$
\int_{B_{\tilde{s}}}\left|\tau_{s, h} D u\right|^{2} \leqslant M^{2}|h|^{2}
$$

and so, by Lemma 2, we get

$$
\|D u\|_{L^{\frac{2 n}{n-2}\left(B_{\tilde{s}}\right)}} \leqslant C\left\{\left[\int_{B_{t}}(k(x)+k(x+h))^{2}(1+|D u|)^{2} \frac{1}{(t-\tilde{s})^{2}} \int_{B_{\lambda r}}|D u|^{2}+\int_{B_{t}}|b(x)|^{2}\right]^{\frac{1}{2}}\right\} .
$$

The a priori ${ }_{2 \Omega}$ sssumption $D^{2} u \in L_{l o c}^{2}(\Omega)$ yields by the Sobolev embedding theorem that $D u \in L_{l o c}^{\frac{n-2}{n-2}}(\Omega)$ and so, by Holder's inequality

$$
\begin{aligned}
\int_{B_{\tilde{s}}}|D u|^{\frac{2 n}{n-2}} \leqslant & C\left[\int_{B_{t}}(k(x)+k(x+h))^{2}(1+|D u|)^{2}\right]^{\frac{n}{n-2}} \\
& +C\left[\frac{1}{(t-\tilde{s})^{2}} \int_{B_{\lambda r}}|D u|^{2}\right]^{\frac{n}{n-2}}+C\left[\int_{B_{t}}|b(x)|^{2}\right]^{\frac{n}{n-2}} \\
\leqslant & {\left[C\left(\int_{B_{\lambda r}} k^{n}(x)\right)^{\frac{2}{n}}\left(\int_{B_{t}}(1+|D u|)^{\frac{2 n}{n-2}}\right)^{\frac{n-2}{n}}\right]^{\frac{n}{n-2}} } \\
& +\left[\frac{C}{(t-\tilde{s})^{2}} \int_{B_{\lambda r}}|D u|^{2}\right]^{\frac{n}{n-2}}+C\left[\int_{B_{t}}|b(x)|^{2}\right]^{\frac{n}{n-2}} \\
\leqslant & C\left(\int_{B_{\lambda r}} k^{n}(x) d x\right)^{\frac{2}{n-2}}\left(\int_{B_{t}}(1+|D u|)^{\frac{2 n}{n-2}}\right) \\
& \left.+\frac{C}{(t-\tilde{s})^{\frac{2 n}{n-2}}}\left(\int_{B_{\lambda r}}|D u|^{2}\right)^{\frac{n}{n-2}}+C\left(\int_{B_{t}}|b(x)|^{2}\right)\right)^{\frac{n}{n-2}}
\end{aligned}
$$

where $C=C(\alpha, \beta, Q)$.

By the absolute continuity of the integral there exists $R_{0}$ such that

$$
C\left(\int_{B_{R_{0}}} k^{n}(x)\right)^{\frac{2}{n-2}}<\frac{1}{2} .
$$


Hence, choosing $R<R_{0}$ determined in (3.10), estimate (3.9) becomes

$$
\begin{aligned}
\int_{B_{\tilde{s}}}|D u|^{\frac{2 n}{n-2}} \leqslant & \frac{1}{2} \int_{B_{t}}(1+|D u|)^{\frac{2 n}{n-2}}+\frac{C}{(t-\tilde{s})^{\frac{2 n}{n-2}}}\left(\int_{B_{\lambda r}}|D u|^{2}\right)^{\frac{n}{n-2}} \\
& +C\left(\int_{B_{t}}|b(x)|^{2}\right)^{\frac{n}{n-2}}
\end{aligned}
$$

and so

$$
\begin{aligned}
\int_{B_{\tilde{s}}}|D u|^{\frac{2 n}{n-2}} \leqslant & \frac{1}{2} \int_{B_{t}}|D u|^{\frac{2 n}{n-2}}+\frac{C}{(t-\tilde{s})^{\frac{2 n}{n-2}}}\left(\int_{B_{\lambda r}}|D u|^{2}\right)^{\frac{n}{n-2}} \\
& +C\left(\int_{B_{\lambda r}}|b(x)|^{2}\right)^{\frac{n}{n-2}}+C R^{n}
\end{aligned}
$$

Since the previous inequality is valid for all radii $r<\tilde{s}<t<\lambda r$, by iteration Lemma 3, with

$$
\begin{aligned}
& h(s)=\int_{B_{s}}|D u|^{\frac{2 n}{n-2}}, A=\left(\int_{B_{\lambda r}}|D u|^{2}\right)^{\frac{n}{n-2}}, \\
& B=\left(\int_{B_{\lambda r}}|b|^{2}\right)^{\frac{n}{n-2}}+C R^{n}
\end{aligned}
$$

we deduce that:

$$
\begin{gathered}
\int_{B_{r}}|D u|^{\frac{2 n}{n-2}} \leqslant \frac{C}{(\lambda r-r)^{\frac{2 n}{n-2}}}\left(\int_{B_{\lambda r}}|D u|^{2}\right)^{\frac{n}{n-2}}+C\left(\int_{B_{\lambda r}}|b(x)|^{2}\right)^{\frac{n}{n-2}}+ \\
+C R^{n} .
\end{gathered}
$$

On the other hand, choosing a cut off function such that $\rho=1$ on $B_{\frac{R}{2}}, \rho \in C_{0}^{\infty}(B r)$, $|D \rho| \leqslant \frac{C}{r-\frac{R}{2}}$ and arguing again as we did from (3.1) to (3.7) we obtain that

$$
\int_{B_{\frac{R}{2}}} \frac{\left|\tau_{s, h} D u\right|^{2}}{|h|^{2}} \leqslant C\left|h^{2}\right|\left[\left(\int_{B_{r}}|D u|^{\frac{2 n}{n-2}}\right)^{\frac{n-2}{n}}+\frac{1}{\left(r-\frac{R}{2}\right)^{2}} \int_{B_{R}}|D u|^{2}+\int_{B_{r}}|b|^{2}\right] .
$$

Therefore, by estimate (3.11) we get

$$
\int_{B_{\frac{R}{2}}} \frac{\left|\tau_{s, h} D u\right|^{2}}{|h|^{2}} \leqslant C|h|^{2}\left[\int_{B_{R}}|D u|^{2}+\int_{B_{R}}|b|^{2}\right],
$$

and so, by Lemma 2, we conclude with 


$$
\int_{B_{\frac{R}{2}}}\left|D^{2} u\right|^{2} \leqslant C\left(\int_{B_{R}} 1+|D u|^{2}+\int_{B_{R}}|b|^{2}\right)
$$

where $C=C(\alpha, \beta, Q, n, R)$.

Step 2. The approximation Fix a compact set $\Omega^{\prime} \subset \Omega$, and for a smooth kernel $\phi \in C_{c}^{\infty}\left(B_{1}(0)\right)$, with $\phi \geqslant 0$ and $\int_{B_{1}(0)} \phi=1$. Let us consider the corresponding family of mollifiers $\left(\phi_{\varepsilon}\right)_{\varepsilon>0}$ and set

$$
\begin{aligned}
& f_{\varepsilon}=f * \phi_{\varepsilon} \\
& b_{\varepsilon}=b * \phi_{\varepsilon} \\
& k_{\varepsilon}=k * \phi_{\varepsilon} \\
& A_{\varepsilon}(x, \xi)=\int_{B_{1}} \phi(\omega) A(x+\varepsilon \omega, \xi) d \omega
\end{aligned}
$$

on $\Omega^{\prime}$, for each positive $\varepsilon<\operatorname{dist}\left(\Omega^{\prime}, \Omega\right)$. It can be easy checked that the assumptions (1.2)-(1.5) imply the followings

$$
\begin{gathered}
\alpha|\xi-\eta|^{2} \leqslant\left\langle A_{\varepsilon}(x, \xi)-A_{\varepsilon}(x, \eta), \xi-\eta\right\rangle, \\
\left|A_{\varepsilon}(x, \xi)-A_{\varepsilon}(x, \eta)\right| \leqslant \beta|\xi-\eta|, \\
\left|f_{\varepsilon}(x)\right| \leqslant Q b_{\varepsilon}(x) .
\end{gathered}
$$

Furthermore

$$
\left|A_{\varepsilon}(x, \xi)-A_{\varepsilon}(y, \xi)\right| \leqslant\left(k_{\varepsilon}(x)+k_{\varepsilon}(y)\right)|x-y|(1+|\xi|)
$$

where $k_{\varepsilon}(x)$ is defined in (3.13).

Let $u \in W_{0}^{1,2}(\Omega)$ be the solution of (1.1), let us fix a ball $B_{R} \subset \Omega^{\prime}$ and let us denote by $u_{\varepsilon} \in W^{1,2}\left(B_{R}\right)$ the unique solution of the Dirichlet problem

$$
\begin{cases}\operatorname{div} A_{\varepsilon}(x, D v)+b_{\varepsilon}(x) v=f_{\varepsilon} & \text { in } B_{R} \\ v=u & \text { on } \partial B_{R} .\end{cases}
$$

Note that, by the classical theory, we have $u_{\varepsilon} \in W_{l o c}^{2,2}\left(B_{R}\right)$ for each $\varepsilon>0$ (see for example [10]).

Our first aim is to prove that, for every $\varepsilon>0$, on $B_{R}$ we have

$$
\left|u_{\varepsilon}\right| \leqslant Q \text {. }
$$

To this purpose, let us set

$$
w^{\varepsilon}= \begin{cases}u_{\varepsilon} & \text { in }\left\{u_{\varepsilon} \leqslant Q\right\} \cap B_{R}, \\ Q & \text { in }\left\{u_{\varepsilon}>Q\right\} \cap B_{R} .\end{cases}
$$

Since $|u| \leqslant Q$ on $\partial B_{R}$ then $w^{\varepsilon}=u_{\varepsilon}=u$ on $\partial B_{R}$, and hence $w^{\varepsilon}$ is an admissible test function for problem (3.18), i.e. 


$$
\int_{B_{R}}\left\langle A_{\varepsilon}\left(x, D u_{\varepsilon}\right), D w^{\varepsilon}\right\rangle+\int_{B_{R}} b_{\varepsilon} w^{\varepsilon} u_{\varepsilon}=\int_{B_{R}} f_{\varepsilon} w^{\varepsilon} .
$$

By the definition of $w^{\varepsilon}$, we get

$$
\begin{aligned}
& \int_{B_{R} \cap\left\{u_{\varepsilon} \leqslant Q\right\}}\left\langle A_{\varepsilon}\left(x, D u_{\varepsilon}\right), D u_{\varepsilon}\right\rangle \\
& +\int_{\left\{u_{\varepsilon} \leqslant Q\right\}} b_{\varepsilon} u_{\varepsilon}^{2}+\int_{\left\{u_{\varepsilon}>Q\right\}} b_{\varepsilon} u_{\varepsilon} Q \\
& \quad=\int_{\left\{u_{\varepsilon} \leqslant Q\right\}} f_{\varepsilon} u_{\varepsilon}+\int_{\left\{u_{\varepsilon}>Q\right\}} f_{\varepsilon} Q .
\end{aligned}
$$

Since also $u_{\varepsilon}$ is an admissible test function for the equation in (3.18) then we have

$$
\int_{B_{R}}\left\langle A_{\varepsilon}\left(x, D u_{\varepsilon}\right), D u_{\varepsilon}\right\rangle+\int_{B_{R}} b_{\varepsilon} u_{\varepsilon}^{2}=\int_{B_{R}} f_{\varepsilon} u_{\varepsilon} .
$$

Subtracting (3.21) to (3.20) and by using (3.16), we get

$$
\begin{aligned}
\int_{B_{R} \cap\left\{u_{\varepsilon}>Q\right\}}\left\langle A_{\varepsilon}\left(x, D u_{\varepsilon}\right), D u_{\varepsilon}\right\rangle=\int_{\left\{u_{\varepsilon}>Q\right\}} b_{\varepsilon} u_{\varepsilon}\left(Q-u_{\varepsilon}\right) \\
\quad+\int_{\left\{u_{\varepsilon}>Q\right\}} f_{\varepsilon}\left(u_{\varepsilon}-Q\right)=\int_{\left\{u_{\varepsilon}>Q\right\}}\left(f_{\varepsilon}-b_{\varepsilon} u_{\varepsilon}\right)\left(u_{\varepsilon}-Q\right) \\
\leqslant \\
\int_{\left\{u_{\varepsilon>Q}\right\}} b_{\varepsilon}\left(Q-u_{\varepsilon}\right)\left(u_{\varepsilon}-Q\right) \\
=-\int_{\left\{u_{\varepsilon}>Q\right\}} b_{\varepsilon}\left(u_{\varepsilon}-Q\right)^{2} \leqslant 0 .
\end{aligned}
$$

Previous inequality combined with (3.14) gives

$$
\alpha \int_{\left\{u_{\varepsilon}>Q\right\}}\left|D u_{\varepsilon}\right|^{2} \leqslant 0
$$

which implies $u_{\varepsilon} \leqslant Q$ a.e. in $B_{R}$, as desired. Arguing analogously we can prove $u_{\varepsilon} \geqslant-Q$ in $B_{R}$, hence we can conclude with (3.19).

Now we want to prove that the sequence $\left(D u_{\varepsilon}\right)_{\varepsilon}$ is bounded in $L^{2}$.

To this aim using $u_{\varepsilon}$ as test function in (3.18), by (3.14), we get 


$$
\begin{aligned}
\alpha \int_{B_{R}}\left|D u_{\varepsilon}\right|^{2} & \leqslant \int_{B_{R}}\left\langle A_{\varepsilon}\left(x, D u_{\varepsilon}\right), D u_{\varepsilon}\right\rangle \\
& =\int_{B_{R}} f_{\varepsilon} u_{\varepsilon}-\int_{B_{R}} b_{\varepsilon} u_{\varepsilon} u_{\varepsilon} \\
& \leqslant \int_{B_{R}}\left|f_{\varepsilon}\right|\left|u_{\varepsilon}\right|+\int_{B_{R}} b_{\varepsilon}\left|u_{\varepsilon}\right|^{2} .
\end{aligned}
$$

By using (3.19) and (3.16), we obtain

$$
\alpha \int_{B_{R}}\left|D u_{\varepsilon}\right|^{2} \leqslant Q \int_{B_{R}}\left|f_{\varepsilon}\right|+Q^{2} \int_{B_{R}} b_{\varepsilon} \leqslant 2 Q^{2} \int_{B_{R}} b_{\varepsilon} .
$$

Since $b_{\varepsilon} \rightarrow b$ strongly in $L^{2}$

$$
\alpha \int_{B_{R}}\left|D u_{\varepsilon}\right|^{2} \leqslant C\left(Q,\|b\|_{2}\right)
$$

then

$$
\int_{B_{R}}\left|D u_{\varepsilon}\right|^{2} \leqslant C\left(\alpha, Q,\|b\|_{2}\right)
$$

as we wanted.

The boundedness of $\left(D u_{\varepsilon}\right)_{\varepsilon}$ in $W^{1,2}$ implies that there exists $v \in W^{1,2}\left(B_{R}\right)$ such that $D u_{\varepsilon} \rightarrow D v$ in $W^{1,2}\left(B_{R}\right)$ and, since $u_{\varepsilon}=u$ on $\partial B_{R}$ in the sense of the trace, we also have $v=u$ on $\partial B_{R}$.

Our next aim is to prove $D u_{\varepsilon} \rightarrow D v$ strongly in $L_{l o c}^{2}\left(B_{R}\right)$.

Since $u_{\varepsilon}$ is a solution of (3.18), it is well known that $u_{\varepsilon} \in W_{l o c}^{2,2}\left(B_{R}\right)$, and, since $\left|f_{\varepsilon}\right|<Q b_{\varepsilon}$ and $\left\|u_{\varepsilon}\right\|_{\infty} \leqslant Q$, we can apply the a priori estimate in (3.12) to each $u_{\varepsilon}$, thus getting

$$
\int_{B_{r}}\left|D^{2} u_{\varepsilon}\right|^{2} \leqslant C \int_{B_{R}}\left(1+\left|D u_{\varepsilon}\right|^{2}\right)+C \int_{B_{R}}\left|b_{\varepsilon}\right|^{2} \leqslant C\left(\alpha, Q,\|b\|_{2}\right)
$$

for every $B_{r}, r<R$. This implies $u_{\varepsilon} \rightarrow v$ weakly in $W_{l o c}^{2,2}\left(B_{R}\right)$ and then $u_{\varepsilon} \rightarrow v$ strongly in $W_{l o c}^{1,2}\left(B_{R}\right)$.

Now we want to show that $v$ is local solution of the equation in (1.1).

Since $u_{\varepsilon}$ is a solution of (3.18), we have

$$
\begin{aligned}
\int_{B_{R}}\langle A(x, D v), D \varphi\rangle & =\int_{B_{R}}\left\langle A_{\varepsilon}\left(x, D u_{\varepsilon}\right)-A_{\varepsilon}\left(x, D u_{\varepsilon}\right)+A(x, D v), D \varphi\right\rangle \\
& =\int_{B_{R}}\left\langle A(x, D v)-A_{\varepsilon}\left(x, D u_{\varepsilon}\right), D \varphi\right\rangle+\int_{B_{R}}\left(f_{\varepsilon} \varphi-b_{\varepsilon} u_{\varepsilon} \varphi\right) \\
& =I_{1, \varepsilon}+I_{2, \varepsilon},
\end{aligned}
$$


for every $\varphi \in C_{0}^{\infty}\left(B_{R}\right)$. Let us estimate $I_{1, \varepsilon}$ and $I_{2, \varepsilon}$ separately. In order to estimate $I_{1, \varepsilon}$ we use assumption (1.3) and the definition of $A_{\varepsilon}(x, \xi)$ as follows

$$
\begin{aligned}
\left|I_{1, \varepsilon}\right| \leqslant & \int_{B_{R}}\left|A(x, D v)-A_{\varepsilon}\left(x, D u_{\varepsilon}\right)\right||D \varphi| \\
\leqslant & \int_{B_{R}}\left|A(x, D v)-A\left(x, D u_{\varepsilon}\right)\right||D \varphi| \\
& +\int_{B_{R}}\left|A\left(x, D u_{\varepsilon}\right)-A_{\varepsilon}\left(x, D u_{\varepsilon}\right)\right||D \varphi| \\
\leqslant & \beta \int_{B_{R}}\left|D v-D u_{\varepsilon}\right||D \varphi| \\
& +\int_{B_{R}}\left|A\left(x, D u_{\varepsilon}\right)-\int_{B_{R}} \phi(\omega) A\left(x+\varepsilon \omega, D u_{\varepsilon}\right) d \omega\right||D \varphi| \\
\leqslant & \beta \int_{B_{R}}\left|D v-D u_{\varepsilon}\right||D \varphi| \\
& +\|D \varphi\|_{\infty} \int_{B_{R}}\left|\int \phi(\omega)\left[A\left(x+\varepsilon \omega, D u_{\varepsilon}\right)-A\left(x, D u_{\varepsilon}\right)\right] d \omega\right| .
\end{aligned}
$$

Now, using assumption (1.4), in the second integral in the right hand side, we obtain

$$
\begin{aligned}
\left|I_{1, \varepsilon}\right| \leqslant & \beta\|D \varphi\|_{\infty} \int_{\operatorname{supp} \varphi}\left|D v-D u_{\varepsilon}\right| \\
& +\varepsilon\|D \varphi\|_{\infty} \cdot \int_{B_{R}}\left(\int_{B_{R}} \phi(\omega)|k(x+\varepsilon \omega)+k(x)|\left(1+\left|D u_{\varepsilon}\right|\right) d \omega\right) d x \\
\leqslant & \beta\|D \varphi\|_{\infty} \int_{\operatorname{supp} \varphi}\left|D v-D u_{\varepsilon}\right|+\varepsilon\|D \varphi\|_{\infty} \int_{B_{R}}\left(k_{\varepsilon}+k\right)\left(1+\left|D u_{\varepsilon}\right|\right) \\
\leqslant & \beta\|D \varphi\|_{\infty} \int_{\operatorname{supp} \varphi}\left|D v-D u_{\varepsilon}\right|+\varepsilon\|D \varphi\|_{\infty}\left\|k_{\varepsilon}+k\right\|_{2}\left(\left\|D u_{\varepsilon}\right\|_{2}+c_{n}\left|B_{R}\right|\right) .
\end{aligned}
$$

Since $\left\|k_{\varepsilon}\right\|_{L^{2}\left(B_{R}\right)} \leqslant C$ and $D u_{\varepsilon} \rightarrow D v$ strongly in any compact subset of $B_{R}$, then

$$
\lim _{\varepsilon \rightarrow 0} I_{1, \varepsilon}=0 \text {. }
$$

Next, we have 


$$
\begin{aligned}
\left|I_{2, \varepsilon}-\int_{B_{R}}(f-b v) \varphi\right|= & \left|\int_{\operatorname{supp} \varphi}\left[\left(f_{\varepsilon}-b_{\varepsilon} u_{\varepsilon}\right) \varphi-(f-b v) \varphi\right]\right| \\
\leqslant & \int_{\operatorname{supp} \varphi}\left|f_{\varepsilon}-f\right| \varphi+\int_{\operatorname{supp} \varphi}\left|b_{\varepsilon} u_{\varepsilon}-b v \| \varphi\right| \\
\leqslant & \int_{\operatorname{supp} \varphi}\left|f_{\varepsilon}-f\left\|\varphi\left|+\int_{\operatorname{supp} \varphi}\right| b_{\varepsilon}-b|| u_{\varepsilon}\right\| \varphi\right| \\
& +\int_{\operatorname{supp} \varphi}|b|\left|v-u_{\varepsilon} \| \varphi\right| \\
& \leqslant\|\|_{\infty} \int_{B_{R}}\left|f_{\varepsilon}-f\right|+\|\varphi\|_{\infty} Q \int_{B_{R}}\left|b_{\varepsilon}-b\right| \\
& +\|\varphi\|_{\infty}\left(\int_{B_{R}}|b|^{2}\right)^{\frac{1}{2}}\left(\int_{\operatorname{supp} \varphi}\left|v-u_{\varepsilon}\right|^{2}\right)^{\frac{1}{2}},
\end{aligned}
$$

where we used (3.19).

Since

$$
\begin{aligned}
f_{\varepsilon} & \rightarrow f \text { strongly in } L^{2}\left(B_{R}\right) \\
b_{\varepsilon} & \rightarrow b \text { strongly in } L^{2}\left(B_{R}\right) \\
u_{\varepsilon} & \rightarrow v \text { strongly in } L_{l o c}^{2}\left(B_{R}\right)
\end{aligned}
$$

Then

$$
\lim _{\varepsilon \rightarrow 0} I_{2, \varepsilon}=\int_{B_{R}}(f-b v) \varphi .
$$

Hence passing to the limit as $\varepsilon \rightarrow 0$ in (3.22), we get

$$
\int_{B_{R}}\langle A(x, D v), D \varphi\rangle=\int_{B_{R}}-b v \varphi+\int_{B_{R}} f \varphi, \quad \forall \varphi \in C_{0}^{\infty}(\Omega),
$$

i.e. $v$ is a solution to the equation in (1.1).

Finally we want to show that $v=u$.

Since $v=u$ on $\partial B_{R}$, then $v-u$ is an admissible function test, for the equation in the problem (3.18) and also for (1.1) we have

$$
\begin{aligned}
& \int_{B_{R}}\langle A(x, D v), D u-D v\rangle+\int_{B_{R}} b v(u-v)=\int_{B_{R}} f(u-v) \\
& \int_{B_{R}}\langle A(x, D u), D u-D v\rangle+\int_{B_{R}} b u(u-v)=\int_{B_{R}} f(u-v)
\end{aligned}
$$

Subtracting (3.23) to (3.24) we get 


$$
\int_{B_{R}}\langle A(x, D u)-A(x, D v), D u-D v\rangle=-\int_{B_{R}} b(u-v)^{2} \leqslant 0 .
$$

On the other hand, by (3.14) it holds that

$$
\alpha \int_{B_{R}}|D u-D v|^{2} \leqslant \int_{B_{R}}\langle A(x, D u)-A(x, D v), D u-D v\rangle \leqslant 0,
$$

i.e.

$$
u=v \quad \text { a.e. in } B_{R} \text {. }
$$

Definitively we proved that $u_{\varepsilon} \rightarrow u$ in $W_{l o c}^{2,2}\left(B_{R}\right)$ then $u_{\varepsilon} \rightarrow u$ strongly in $W_{l o c}^{1,2}\left(B_{R}\right)$, hence we can conclude the proof, simply by passing to limit in (3.12), since the operator $A_{\varepsilon}(x, \xi)$ satisfies (3.14)-(3.17).

Proof of Corollary 1. It is sufficient to combine inequality in Theorem 1 with Lemma 4 for $p=2$ contained in Ref. [9].

\section{Example}

Let us consider the function $u(x)=|x|^{-\gamma}-1$ where $x \in B=B(0,1)$ and $\gamma>0$, obviously, $u(x) \notin L^{\infty}(B)$.

By setting, $u(B)=\varphi(|B|), u(x)=\varphi(\rho)=\rho^{-\gamma}$, it is well known that

$$
\Delta u=\varphi^{\prime \prime}(\rho)+\frac{n-1}{\rho} \varphi^{\prime}(\rho)
$$

where $\varphi^{\prime}(\rho)=-\gamma \rho^{-\gamma-1}$ and $\varphi^{\prime \prime}(\rho)=\gamma(\gamma+1) \rho^{-\gamma-2}$.

Hence, for $\gamma>0 u(x)$ solves problem (1.1) for

$$
b(x)=\frac{\gamma n}{|x|^{2}} \quad \text { and } \quad f(x)=\frac{\gamma(\gamma+2)}{|x|^{\gamma+2}}+\frac{\gamma n}{\rho^{2}} .
$$

Clearly, for such $b(x)$ and $f(x)$, condition (1.5) is not satisfied.

Moreover

$$
\int_{B}|D u|^{2} d x \approx \int_{B(0,1)} \frac{1}{|x|^{2 \gamma+2}} d x \approx \int_{0}^{1} \frac{1}{\rho^{2 \gamma+2}} \rho^{n-1} d \rho \approx\left[\rho^{n-2 \gamma-2}\right]_{0}^{1}
$$

and

$$
\int_{B}\left|D^{2} u\right|^{2} d x \approx \int \frac{1}{|x|^{2 \gamma+4}} d x \approx \int_{0}^{1} \frac{1}{\rho^{2 \gamma+4}} \rho^{n-1} d \rho \approx\left[\rho^{n-2 \gamma-4}\right]_{0}^{1}
$$

are finite for $\gamma<\frac{n}{2}-1$ and $\gamma<\frac{n}{2}-2$, respectively.

Hence, for $\gamma \in\left(\frac{n}{2}-2, \frac{n}{2}-1\right)$, we have that $u(x) \in W^{1,2}(B)$ but $u(x) \notin W^{2,2}(B)$. 
This example shows that Theorem 1 can be false if condition (1.5) does not hold.

We also want to highlight that condition (1.5) cannot be avoided even assuming more integrability for $b(x)$ and $f(x)$ than $L^{2}$.

Indeed

$$
\int_{B(0,1)}|b(x)|^{p} d x \approx \int_{B(0,1)} \frac{1}{|x|^{2 p}} d x \approx \int_{0}^{1} \frac{1}{\rho^{2 p}} \rho^{n-1} d \rho \approx\left[\rho^{n-2 p}\right]_{0}^{1}
$$

and

$$
\int_{B(0,1)}|f(x)|^{p} d x \lesssim \int_{B(0,1)} \frac{1}{|x|^{(\gamma+2) p}} d x \approx \int_{0}^{1} \frac{1}{\rho^{(\gamma+2) p}} \rho^{n-1} d \rho \approx\left[\rho^{n-(\gamma+2) p}\right]_{0}^{1}
$$

are finite for $p<\frac{n}{2}$ and $p<\frac{n}{\gamma+2}$, respectively.

Actually $b(x)$ and $f(x)$ belong to $L^{p}$ for all $p<\frac{n}{\gamma+2}$, nevertheless $u(x) \notin W^{2,2}$, hence Theorem 1 is not true.

Acknowledgements Open access funding provided by Università degli Studi di Napoli Federico II within the CRUI-CARE Agreement.

Open Access This article is licensed under a Creative Commons Attribution 4.0 International License, which permits use, sharing, adaptation, distribution and reproduction in any medium or format, as long as you give appropriate credit to the original author(s) and the source, provide a link to the Creative Commons licence, and indicate if changes were made. The images or other third party material in this article are included in the article's Creative Commons licence, unless indicated otherwise in a credit line to the material. If material is not included in the article's Creative Commons licence and your intended use is not permitted by statutory regulation or exceeds the permitted use, you will need to obtain permission directly from the copyright holder. To view a copy of this licence, visit http://creativecommons.org/licen ses/by/4.0/.

\section{References}

1. Arcoya, D., Boccardo, L.: Regularizing effect of the interplay between coefficients in some elliptic equations. J. Funct. Anal. 208, 1153-1266 (2015)

2. Arcoya, D., Boccardo, L.: Regularizing effect of $L^{q}$ interplay between coefficients in some elliptic equations. Journal de Mathématiques Pures et Appliquées (2020). https://doi.org/10.1016/j.matpu r.2017.08.001

3. Alvino, A., Trombetti, G.: Second order elliptic equations whose coefficients have their first derivatives weakly $L^{n}$. Ann. Mat. Pura Appl. 138(4), 331-340 (1984)

4. Capone, C., Fiorenza, A., Kalamajska, A.: Strongly nonlinear Gagliardo-Nirenberg inequality in Orlicz spaces and Boyd indices, Atti Accad. Naz. Lincei Rend. Lincei Mat. Appl. 28(1), 119-141 (2017)

5. Clop, A., Giova, R., Passarelli di Napoli, A.: Besov regularity for solutions of $p$-harmonic equations. https://doi.org/10.1515/anona-2017-0030

6. Carozza, M., Kristensen, J., Passarelli di Napoli, A.: Higher differentiability of minimizers of convex variational integrals. Annales Inst. H. Poincaré (C) Non Linear Analysis 28(3), 395-411 (2011)

7. Fiorenza, A., Sbordone, C.: Existence and uniqueness results for solutions of nonlinear equations with right-hand side in $L^{1}$. Studia Math. 127(3), 223-231 (1998) 
8. Giova, R.: Higher differentiability for $n$-harmonic systems with Sobolev coefficients. J. Differ. Equ. 259, 5667-5687 (2015)

9. Giova, R., Passarelli di Napoli, A.: Regularity results for a priori bounded minimizers of non-autonomous functionals with discontinuous coefficients. Adv. Calc. Var. (2020). https://doi.org/10.1515/ acv-2016-0059

10. Giusti, E.: Direct Method in the Calculus of Variations. World Scientific, Singapore (2003)

11. Greco, L., Moscariello, G., Radice, T.: Nondivergence elliptic equations with unbounded coefficients. Discrete Contin. Dyn. Syst. 11, 43-55 (2009)

12. Hajlasz, P.: Sobolev spaces on an arbitrary metric space. Potential Anal. 5, 403-415 (1996)

13. Iwaniec, T., Sbordone, C.: Riesz transform and elliptic PDE's with VMO coefficients. J. d'Analyse Math. 74, 183-212 (1998)

14. Iwaniec, T., Sbordone, C.: Quasiharmonic fields, Annales de l'I.H.P. Analyse non linéaire 18(5), 519-572 (2001)

15. Miranda, C.: Sulle equazioni ellittiche del secondo ordine di tipo non variazionale con coefficienti non discontinui. Ann. Mat. Pura Appl. 4(63), 353-386 (1963)

16. Passarelli di Napoli, A.: Higher differentiability of minimizers of variational integrals with Sobolev coefficients. Adv. Calc. Var. 7(1), 59-89 (2014)

17. Passarelli di Napoli, A.: Higher differentiability of solutions of elliptic systems with Sobolev coefficients: the case $p=n=2$. Potential Anal. 41(3), 715-735 (2014)

18. Radice, T.: A higher integrability result for nondivergence elliptic equations. Ann. Mat. Pura Appl. 187, 93-103 (2008)

19. Radice, T.: Regularity result for nondivergence elliptic equations with unbounded coefficients. Differ. Integral Equ. 23(9-10), 989-1000 (2010)

20. Radice, T., Zecca, G.: Existence and uniqueness for nonlinear elliptic equations with unbounded coefficients. Ric. Mat. 63(2), 355-367 (2014)

Publisher's Note Springer Nature remains neutral with regard to jurisdictional claims in published maps and institutional affiliations. 